\title{
Energetic Properties, Spectroscopy, and Reactivity of $\mathrm{NF}_{3} \mathrm{O}$
}

Monica Vasiliu, ${ }^{1}$ Tarek Trabelsi, ${ }^{2}$ Joseph S. Francisco, ${ }^{2}$ Karl O. Christe, ${ }^{3}$ and David A. Dixon ${ }^{1, * * *}$

${ }^{1}$ Department of Chemistry and Biochemistry, Shelby Hall, The University of Alabama, Box 870336, Tuscaloosa, AL 35487-0336.

${ }^{2}$ Department of Earth and Environmental Science and Department of Chemistry, University of Pennsylvania, Philadelphia, PA 19104-6243

${ }^{3}$ Loker Hydrocarbon Research Institute and Department of Chemistry University of Southern California, Los Angeles, CA 90089-1661

Complete references:

Ref. 32: Frisch, M. J.; Trucks, G. W.; Schlegel, H. B.; Scuseria, G. E.; Robb, M. A.; Cheeseman, J. R.; Scalmani, G.; Barone, V.; Petersson, G. A.; Nakatsuji, H.; Li, X.; Caricato, M.; Marenich, A. V.; Bloino, J.; Janesko, B. G.; Gomperts, R.; Mennucci, B.; Hratchian, H. P.; Ortiz, J. V.; Izmaylov, A. F.; Sonnenberg, J. L.; Williams-Young, D.; Ding, F.; Lipparini, F.; Egidi, F.; Goings, J.; Peng, B.; Petrone, A.; Henderson, T.; Ranasinghe, D.; Zakrzewski, V. G.; Gao, J.; Rega, N.; Zheng, G.; Liang, W.; Hada, M.; Ehara, M.; Toyota, K.; Fukuda, R.; Hasegawa, J.; Ishida, M.; Nakajima, T.; Honda, Y.; Kitao, O.; Nakai, H.; Vreven, T.; Throssell, K.; Montgomery, J. A., Jr.; Peralta, J. E.; Ogliaro, F.; Bearpark, M. J.; Heyd, J. J.; Brothers, E. N.; Kudin, K. N.; Staroverov, V. N.; Keith, T. A.; Kobayashi, R.; Normand, J.; Raghavachari, K.; Rendell, A. P.; Burant, J. C.; Iyengar, S. S.; Tomasi, J.; Cossi, M.; Millam, J. M.; Klene, M.; Adamo, C.; Cammi, R.; Ochterski, J. W.; Martin, R. L.; Morokuma, K.; Farkas, O.; Foresman, J. B.; Fox, D. J. Gaussian 16, Revision A.03, Gaussian, Inc., Wallingford CT, 2016.

Ref. 52: Werner H.-J.; Knowles, P. J.; Knizia, G.; Manby, F. R.; Schütz, M.; Celani, P.; Györffy, W.; Kats, T.; Korona, T.; Lindh, R.; Mitrushenkov, A.; Rauhut, G.; Shamasundar K. R.; Adler, T. B.; Amos, R. D.; Bernhardsson, A.; Berning, A.; Cooper, D. L.; Deegan, M. J. O.; Dobbyn, A. J.; Eckert, F.; Goll, E.; Hampel, C.; Hesselmann, A.; Hetzer, G.; Hrenar, T.; Jansen, G.; Köppl, C.; Liu, Y.; Lloyd, A. W.; Mata, R. A.; May, A. J.; McNicholas, S. J.; Meyer, W.; Mura, M. E.; Nicklass, A.; O'Neill D. P.; Palmieri, P.; Peng, D.; Pflüger, K.; Pitzer, R.; Reiher, M.; Shiozaki, T.; Stoll, H.; Stone, A. J.; Tarroni, R.; Thorsteinsson, T.; Wang, M. MOLPRO, version 2018.1 a package of ab initio programs, See http://www.molpro.net.

*Email: dadixon@ua.edu 
Table S1. Calculated Harmonic Vibrational Frequencies in $\mathrm{cm}^{-1}$ and Infrared Intensities in $\mathrm{km} / \mathrm{mol}$ at the MP2/aVTZ Level.

\begin{tabular}{|l|c|l|l|l|l|r|r|}
\hline $\mathrm{NF}_{4}^{+}$ & & $\mathrm{NF}_{3} \mathrm{O}$ & & $\mathrm{CF}_{3} \mathrm{O}^{-}$ & & $\mathrm{NF}_{3}$ & \\
\hline$v$ & IR I & $v$ & \multicolumn{1}{l|}{$\mathrm{IR} \mathrm{I}$} & $\mathrm{v}$ & $\mathrm{IR} \mathrm{I}$ & Freq & IR I \\
\hline $1196.4\left(\mathrm{t}_{2}\right)$ & 690 & $1884.5\left(\mathrm{a}_{1}\right)$ & 538 & $1641.5(\mathrm{~A})$ & 799 & $505.5(\mathrm{e})$ & 2 \\
\hline $890.2\left(\mathrm{a}_{1}\right)$ & 0 & $848.8(\mathrm{e})$ & 557 & $900.3(\mathrm{E})$ & 812 & $668.8\left(\mathrm{a}_{1}\right)$ & 2 \\
\hline $628.8\left(\mathrm{t}_{2}\right)$ & 11 & $740.4(\mathrm{a})$ & 118 & $793.5(\mathrm{~A})$ & 115 & $923.3(\mathrm{e})$ & 425 \\
\hline $447.7(\mathrm{e})$ & 0.0 & $539.5(\mathrm{a})$ & 2 & $586.4(\mathrm{~A})$ & 1 & $1057.7(\mathrm{a} 1)$ & 32 \\
\hline & & $507.8(\mathrm{e})$ & 136 & $562.1(\mathrm{E})$ & 61 & & \\
\hline & & $397.8(\mathrm{e})$ & 2 & $410.0(\mathrm{E})$ & 0 & & \\
\hline
\end{tabular}


Table S2. Total Energies at the $\operatorname{CCSD}(\mathrm{T})$ Level of Theory in Atomic Units.

\begin{tabular}{|c|c|c|c|c|c|c|c|c|c|}
\hline Molecule & aVDZ & aVTZ & aVQZ & CBS & $\begin{array}{c}\text { awCVTZ- } \\
\text { core }\end{array}$ & $\begin{array}{c}\text { awCVTZ- } \\
\text { valence }\end{array}$ & CV-corr & $\begin{array}{c}\text { awCVTZ- } \\
\text { DK }\end{array}$ & DK-corr \\
\hline $\mathrm{NF}_{4}^{+}\left({ }^{1} \mathrm{~A}_{1}, \mathrm{~T}_{d}\right)$ & -452.609251 & -452.986646 & -453.104982 & -453.171908 & -453.285427 & -453.014959 & -0.270469 & -453.391794 & -0.376836 \\
\hline $\mathrm{NF}_{3} \mathrm{O}\left({ }^{1} \mathrm{~A}_{1}, \mathrm{C}_{3 v}\right)$ & -428.445282 & -428.798440 & -428.910410 & -428.973872 & -429.093580 & -428.825060 & -0.268520 & -429.166779 & -0.341719 \\
\hline${ }^{2} \mathrm{NF}_{2} \mathrm{O}\left({ }^{2} \mathrm{~A}^{\prime}, \mathrm{C}_{s}\right)$ & -328.819670 & -329.087434 & -329.172788 & -329.221215 & -329.321324 & -329.108010 & -0.213314 & -329.362959 & -0.254949 \\
\hline $\mathrm{NF}_{3} \mathrm{OH}^{+}\left({ }^{1} \mathrm{~A}^{\prime}, \mathrm{C}_{s}\right)$ & -428.673551 & -429.031442 & -429.142999 & -429.206017 & -429.325776 & -429.057542 & -0.268235 & -429.399343 & -0.341801 \\
\hline $\mathrm{NF}_{2} \mathrm{O}^{+} \bullet \mathrm{HF}$ & -428.724501 & -429.079797 & -429.192330 & -429.256099 & -429.375152 & -429.106390 & -0.268763 & -429.448081 & -0.341691 \\
\hline $\mathrm{CF}_{3} \mathrm{O}^{-}\left({ }^{1} \mathrm{~A}_{1}, \mathrm{C}_{3 v}\right)$ & -412.152644 & -412.497193 & -412.607705 & -412.670481 & -412.790240 & -412.523617 & -0.266622 & -412.850468 & -0.326851 \\
\hline $\mathrm{CF}_{2} \mathrm{O}^{-}\left({ }^{1} \mathrm{~A}^{\prime}, \mathrm{C}_{s}\right)$ & -312.405815 & -312.663435 & -312.746529 & -312.793781 & -312.894682 & -312.683466 & -0.211216 & -312.923684 & -0.240218 \\
\hline $\mathrm{O}_{2} \mathrm{~F}_{3}^{+}\left({ }^{1} \mathrm{~A}_{1}, \mathrm{C}_{3 v}\right)$ & -448.176981 & -448.538840 & -448.651992 & -448.715951 & -448.836402 & -448.566955 & -0.269447 & -448.932667 & -0.365712 \\
\hline $\mathrm{OSiF}_{3}^{-}\left({ }^{1} \mathrm{~A}_{1}, \mathrm{C}_{3 v}\right)$ & -663.413564 & -663.772656 & -663.887786 & -663.953181 & -664.280093 & -663.801698 & -0.478394 & -664.715321 & -0.913622 \\
\hline $\mathrm{OSiF}_{2}\left({ }^{1} \mathrm{~A}_{1}, \mathrm{C}_{2 v}\right)$ & -563.553318 & -563.825452 & -563.910870 & -563.959189 & -564.270118 & -563.847492 & -0.422626 & -564.674840 & -0.827348 \\
\hline $\mathrm{OSF}_{3}^{+}\left({ }^{1} \mathrm{~A}_{1}, \mathrm{C}_{3 v}\right)$ & -771.311520 & -771.712365 & -771.833574 & -771.901627 & -772.230823 & -771.744450 & -0.486373 & -773.136228 & -1.391778 \\
\hline $\mathrm{OSF}_{2}^{+}\left({ }^{2} \mathrm{~A}^{\prime}, \mathrm{C}_{s}\right)$ & -671.607658 & -671.912276 & -672.003698 & -672.054948 & -672.367592 & -671.936347 & -0.431246 & -673.242612 & -1.306265 \\
\hline $\mathrm{PF}_{4}^{+}\left({ }^{1} \mathrm{~A}_{1}, \mathrm{~T}_{d}\right)$ & -739.297726 & -739.712344 & -739.836892 & -739.906725 & -740.229249 & -739.744864 & -0.484385 & -740.905607 & -1.160742 \\
\hline FNO $\left({ }^{1} \mathrm{~A}^{\prime}, \mathrm{C}_{s}\right)$ & -229.266627 & -229.450615 & -229.509543 & -229.543007 & -229.623064 & -229.465092 & -0.157971 & -229.633298 & -0.168206 \\
\hline
\end{tabular}


Table S3. T1 values from $\operatorname{CCSD}(\mathrm{T}) / \mathrm{aVTZ}$.

\begin{tabular}{|l|c|}
\hline Molecule & T1 \\
\hline $\mathrm{NF}_{3} \mathrm{O}\left({ }^{1} \mathrm{~A}_{1}, \mathrm{C}_{3 v}\right)$ & 0.0182 \\
\hline${ }^{2} \mathrm{NF}_{2} \mathrm{O}\left({ }^{2} \mathrm{~A}^{\prime}, \mathrm{C}_{s}\right)$ & 0.0261 \\
\hline $\mathrm{NF}_{3} \mathrm{OH}^{+}\left({ }^{1} \mathrm{~A}^{\prime}, \mathrm{C}_{s}\right)$ & 0.0160 \\
\hline $\mathrm{NF}_{2} \mathrm{O}^{+} \cdot \mathrm{HF}$ & 0.0189 \\
\hline $\mathrm{CF}_{3} \mathrm{O}^{-}\left({ }^{1} \mathrm{~A}_{1}, \mathrm{C}_{3 v}\right)$ & 0.0144 \\
\hline $\mathrm{CF}_{2} \mathrm{O}^{-}\left({ }^{1} \mathrm{~A}^{\prime}, \mathrm{C}_{s}\right)$ & 0.0225 \\
\hline $\mathrm{O}_{2} \mathrm{~F}_{3}{ }^{+}\left({ }^{1} \mathrm{~A}_{1}, \mathrm{C}_{3 v}\right)$ & 0.0211 \\
\hline $\mathrm{OSiF}_{3}{ }^{-}\left({ }^{1} \mathrm{~A}_{1}, \mathrm{C}_{3 v}\right)$ & 0.0139 \\
\hline $\mathrm{OSiF}_{2}\left({ }^{1} \mathrm{~A}_{1}, \mathrm{C}_{2 v}\right)$ & 0.0168 \\
\hline $\mathrm{OSF}_{3}{ }^{+}\left({ }^{1} \mathrm{~A}_{1}, \mathrm{C}_{3 v}\right)$ & 0.0319 \\
\hline $\mathrm{OSF}_{2}{ }^{+}\left({ }^{2} \mathrm{~A}^{\prime}, \mathrm{C}_{s}\right)$ & 0.0211 \\
\hline $\mathrm{PF}_{4}{ }^{+}\left({ }^{1} \mathrm{~A}_{1}, \mathrm{~T}_{d}\right)$ & 0.0136 \\
\hline $\mathrm{FNO}^{1}\left({ }^{1} \mathrm{~A}^{\prime}, \mathrm{C}_{s}\right)$ & 0.0228 \\
\hline
\end{tabular}

Table S4. Vertical Excitation Energies of $\mathrm{CF}_{2} \mathrm{Cl}_{2}$ in $\mathrm{eV}$ with $\mathrm{nm}$ Values in Parentheses at the EOM-CCSD/aug-cc-pVTZ Level

\begin{tabular}{|l|l|}
\hline States & Excitation Energy \\
\hline${ }^{3} \mathrm{~B}_{1}$ & $6.54(189)$ \\
\hline${ }^{3} \mathrm{~A}_{2}$ & $6.88(180)$ \\
\hline${ }^{3} \mathrm{~B}_{2}$ & $7.16(173)$ \\
\hline${ }^{3} \mathrm{~A}_{1}$ & $7.29(170)$ \\
\hline${ }^{1} \mathrm{~B}_{2}$ & $7.29(170)$ \\
\hline
\end{tabular}




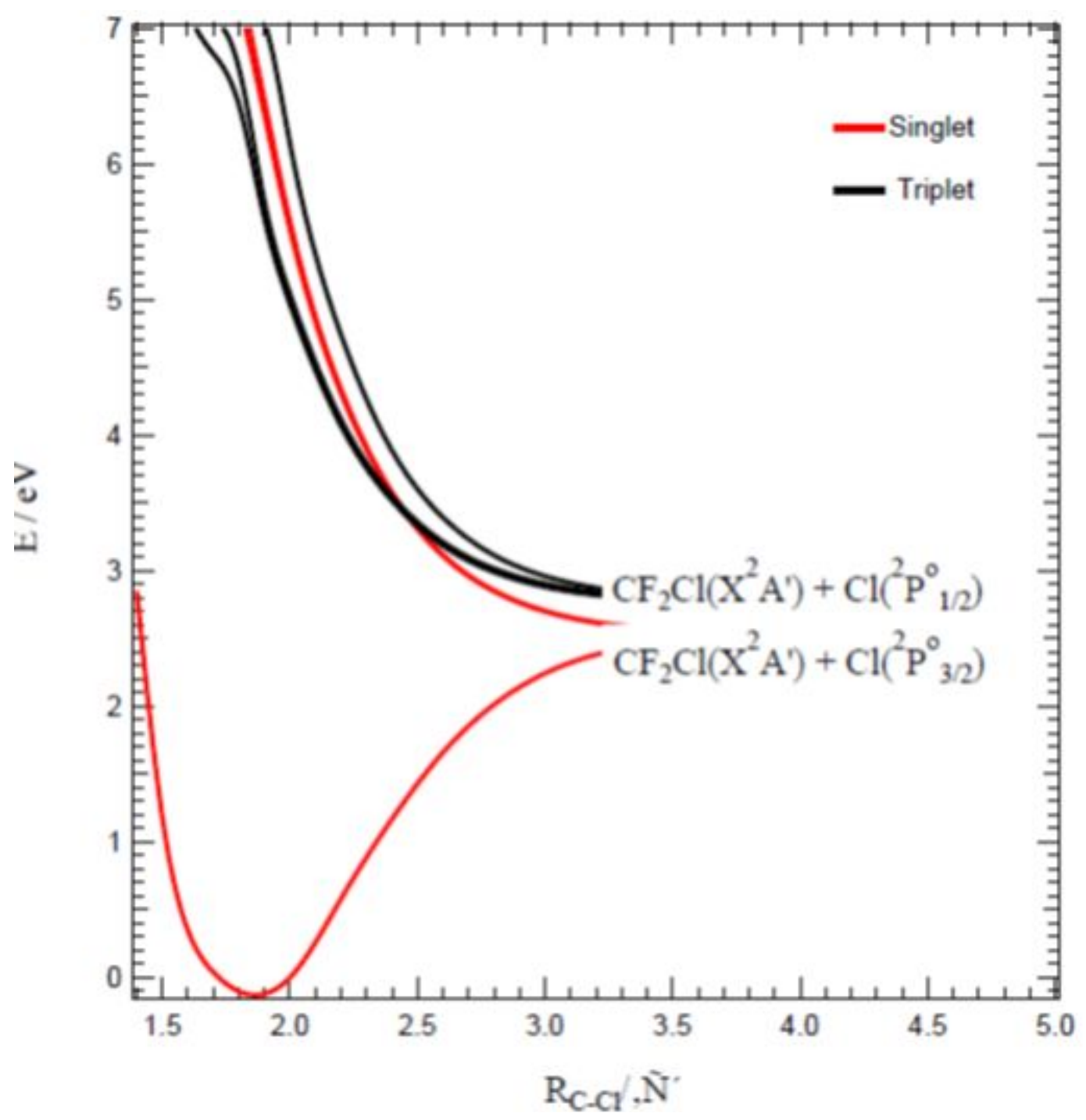

Figure S1. CASSCF/aug-cc-pVTZ one-dimensional PESs of the lowest-lying electronic states of $\mathrm{CF}_{2} \mathrm{Cl}_{2}$ along the $\mathrm{CF}_{2} \mathrm{Cl}-\mathrm{Cl}$ distance 